\title{
Antimicrobial Resistance Pattern and Their Beta-Lactamase Encoding Genes among Pseudomonas aeruginosa Strains Isolated from Cancer Patients
}

\author{
Mai M. Zafer, ${ }^{1}$ Mohamed H. Al-Agamy, ${ }^{2,3}$ Hadir A. El-Mahallawy, \\ Magdy A. Amin, ${ }^{5}$ and Mohammed Seif El-Din Ashour ${ }^{3,6}$ \\ ${ }^{1}$ Department of Microbiology and Immunology, Faculty of Pharmacy, Ahram Canadian University, Cairo, Egypt \\ ${ }^{2}$ Department of Pharmaceutics and Microbiology, College of Pharmacy, King Saud University, P.O. Box 2457, \\ Riyadh 11451, Saudi Arabia \\ ${ }^{3}$ Department of Microbiology and Immunology, Faculty of Pharmacy, Al-Azhar University, Cairo, Egypt \\ ${ }^{4}$ Department of Clinical Pathology, National Cancer Institute, Cairo University, Cairo, Egypt \\ ${ }^{5}$ Department of Microbiology and Immunology, Faculty of Pharmacy, Cairo University, Cairo, Egypt \\ ${ }^{6}$ Department of Microbiology and Immunology, Faculty of Pharmacy, Modern Science and Arts University, \\ 6th October City, Giza, Egypt
}

Correspondence should be addressed to Mohamed H. Al-Agamy; elagamy71@yahoo.com

Received 15 September 2013; Revised 7 January 2014; Accepted 14 January 2014; Published 23 February 2014

Academic Editor: Branka Bedenic

Copyright (C) 2014 Mai M. Zafer et al. This is an open access article distributed under the Creative Commons Attribution License, which permits unrestricted use, distribution, and reproduction in any medium, provided the original work is properly cited.

\begin{abstract}
This study was designed to investigate the prevalence of metallo- $\beta$-lactamases (MBL) and extended-spectrum $\beta$-lactamases (ESBL) in $P$. aeruginosa isolates collected from two different hospitals in Cairo, Egypt. Antibiotic susceptibility testing and phenotypic screening for ESBLs and MBLs were performed on 122 P. aeruginosa isolates collected in the period from January 2011 to March 2012. MICs were determined. ESBLs and MBLs genes were sought by PCR. The resistant rate to imipenem was $39.34 \%$. The resistance rates for $P$. aeruginosa to cefuroxime, cefoperazone, ceftazidime, aztreonam, and piperacillin/tazobactam were $87.7 \%, 80.3 \%, 60.6 \%$, $45.1 \%$, and $25.4 \%$, respectively. Out of 122 P. aeruginosa, $27 \%$ and $7.4 \%$ were MBL and ESBL, respectively. The prevalence of $b l a_{\mathrm{VIM}-2}$, $b l a_{\mathrm{OXA}-10^{-}}, b l a_{\mathrm{VEB}-1}, b l a_{\mathrm{NDM}^{-}}$, and $b l a_{\mathrm{IMP}-1}$-like genes were found in $58.3 \%, 41.7 \%, 10.4 \%, 4.2 \%$, and 2.1\%, respectively. GIM-, SPM-, SIM-, and OXA-2-like genes were not detected in this study. OXA-10-like gene was concomitant with VIM-2 and/or VEB. Twelve isolates harbored both OXA-10 and VIM-2; two isolates carried both OXA-10 and VEB. Only one strain contained OXA-10, VIM2 , and VEB. In conclusion, $b l a_{\mathrm{VIM}-2^{-}}$and $b l a_{\mathrm{OXA}-10^{-}}$-like genes were the most prevalent genes in P. aeruginosa in Egypt. To our knowledge, this is the first report of $b l a_{\mathrm{VIM}-2}, b l a_{\mathrm{IMP}-1}, b l a_{\mathrm{NDM}}$, and $b l a_{\mathrm{OXA}-10}$ in P. aeruginosa in Egypt.
\end{abstract}

\section{Introduction}

Pseudomonas aeruginosa is widely known as an opportunistic organism, frequently involved in infections of immunesuppressed patients, and also causes outbreaks of hospitalacquired infections [1] that cause infections with a high mortality rate [2]. This latter is, in part, attributable to the organism's intrinsically high resistance to many antimicrobials and the development of increased, particularly multidrug, resistance in healthcare settings [3], both of which complicate antipseudomonal chemotherapy. The carbapenems have been the drug of choice for the treatment of infections caused by penicillin or cephalosporin resistant Gram-negative bacilli [4]. However, carbapenem resistance has been observed frequently in nonfermenting bacilli Pseudomonas aeruginosa and Acinetobacter spp. Resistance to carbapenem is due to decreased outer membrane permeability, increased efflux systems, alteration of penicillin-binding proteins, and carbapenem hydrolyzing enzymes-carbapenemase. In the last decade, several classes $\mathrm{A}, \mathrm{B}$, and $\mathrm{D} \beta$-lactamases have been detected in $P$. aeruginosa [5]. The carbapenemases found are mostly metallo- $\beta$-lactamases (MBL), including IMP, VIM, 
SPM, SIM, GIM, AIM, DIM, or NDM enzymes, but serine carbapenemases have also been recorded, including KPC and GES variants [6].

The OXA-ESBLs are mutants of OXA-2 and -10, belonging to class $\mathrm{D}$, whereas the other ESBL belongs to class A. VEB and PER types were found to be the most common (or least rare) ESBL in $P$. aeruginosa in several countries, contrasting to the dominance of CTX-M, SHV, and TEM ESBL in Enterobacteriaceae [7]. Detection of MBL and ESBL producing Gram-negative bacilli especially $P$. aeruginosa is crucial for optimal treatment of patients particularly critically ill and hospitalized patients and to control the spread of resistance. The aim of the present study was phenotypic and genotypic screening for MBL and ESBL producing strains among $P$. aeruginosa isolated from clinical specimens of cancer patients recovered from two hospitals in Cairo, Egypt.

\section{Materials and Methods}

2.1. Bacterial Strains. Hundred twenty-two nonduplicate nonconsecutive $P$. aeruginosa isolates were obtained from clinical specimens submitted for bacteriological testing from hospitalized in-patients admitted to Kasr El Aini Hospital and National Cancer Institute, Cairo University, Egypt, in the period from January 2011 to March 2012. Kasr El Aini School of Medicine and National Cancer Institute are tertiary hospitals belonging to Cairo University, Egypt. The study was approved by Ethics Committee of Cairo University and an informed consent was obtained from all patients receiving treatment and participating in the study. With regard to the specimen site, $P$. aeruginosa were isolated from wound swabs $(n=44)$, blood $(n=29)$, urine $(n=22)$, sputum $(n=11)$, cerebrospinal fluid (CSF) $(n=2)$, genital sites $(n=2)$, catheter tip $(n=2)$, central venous catheter $(n=4)$, ear swab $(n=2)$, pleural tissue specimen $(n=2)$, corneal graft $(n=1)$, and breast abscess $(n=1)$.

2.2. Bacterial Identification. Identification of $P$ aeruginosa was done on the basis of Gram staining, colony morphologies on MacConkey's agar, motility, pigment production, oxidase reaction, growth at $42^{\circ} \mathrm{C}$, and the biochemical tests included in the API 20NE identification kit (Biomerieux, Marcy l'Étoile, France). The Vitek 2 system (Vitek 2 software, version R02.03; Advanced Expert System [AES] software, version R02.00N (bioMerieux, Marcy l'Étoile, France) was used with the ID-GNB card for identification of Gram-negative bacilli. The identified strains were stored in glycerol broth cultures at $-70^{\circ} \mathrm{C}$.

2.3. Antimicrobial Susceptibility Testing. Susceptibility of the isolates to the following antibacterial agents was tested by the Kirby-Bauer disc diffusion method [8] using disks (Oxoid ltd., Basin Stoke, Hants, England) on Mueller Hinton agar and interpreted as recommended by Clinical and Laboratory Standards Institute (CLSI) guidelines [9]: amikacin (AK, $30 \mu \mathrm{g}$ ), aztreonam (ATM, $30 \mu \mathrm{g}$ ), cefepime (FEP, $30 \mu \mathrm{g})$, cefoperazone (CFP, $30 \mu \mathrm{g}$ ), cefotaxime (CTX, $30 \mu \mathrm{g})$, ceftazidime (CAZ, $30 \mu \mathrm{g})$, ceftriaxone
(CRO, $30 \mu \mathrm{g}$ ), cefuroxime (CXM, $30 \mu \mathrm{g}$ ), ciprofloxacin (CIP, $5 \mu \mathrm{g}$ ), imipenem (IPM, $10 \mu \mathrm{g}$ ), meropenem (MEM, $10 \mu \mathrm{g}$ ), piperacillin/tazobactam $(\mathrm{TPZ}, 100 / 10 \mu \mathrm{g})$, polymyxin B (PB, 300 units), and tobramycin (TOB, $10 \mu \mathrm{g})$.

2.4. MIC Determination for $M B L$-Producing P. aeruginosa . The MICs of 9 antibiotics (cefepime, piperacillin/tazobactam, ceftazidime/clavulanic acid, ceftazidime, ciprofloxacin, amikacin, gentamicin, imipenem, and cefotaxime) were determined to $33 P$. aeruginosa isolates that phenotypically produce MBL using Etest (AB Biodisk, Solna, Sweden) as described by the manufacturer. Results were interpreted using CLSI criteria for susceptibility testing [9]. P. aeruginosa ATCC 27853 was used as the reference strain.

2.5. Phenotypic Detection of ESBL. Combined double disc synergy test was performed with discs containing ceftazidime $(30 \mu \mathrm{g})$ alone and in the presence of clavulanate $(10 \mu \mathrm{g})$. In order to inhibit cephalosporinase overproduction, double disc synergy tests were also carried out with the addition of $400 \mu \mathrm{g}$ of boronic acid [10]. Increase in ceftazidime inhibition zone of $\geq 5 \mathrm{~mm}$ in the presence of clavulanic acid as compared with when tested alone was considered to be ESBL producer.

2.6. Phenotypic Detection of $M B L$. A $4 \mu \mathrm{L}$ of $0.5 \mathrm{M}$ EDTA (Sigma Chemicals, St. Louis, MO) was poured on imipenem and ceftazidime disks to obtain a desired concentration of $750 \mu \mathrm{g}$ per disk. The EDTA impregnated antibiotic disks were dried immediately in an incubator and stored at $-20^{\circ} \mathrm{C}$ in air-tight vials without desiccant until used. 0.5 McFarland equivalent overnight broth culture of test strain was inoculated on a plate of Mueller Hinton agar. One $10 \mu \mathrm{g}$ imipenem and one $30 \mu \mathrm{g}$ ceftazidime disks were placed on the agar plate. One each of EDTA impregnated imipenem and ceftazidime disks were also placed on same agar plate. The plate was incubated at $37^{\circ} \mathrm{C}$ for 16 to $18 \mathrm{~h}$. An increase in the zone size of $\geq 7 \mathrm{~mm}$ around the imipenem-EDTA disk or ceftazidimeEDTA compared to imipenem or ceftazidime disks without EDTA was recorded as MBL producing strain [11].

2.7. Preparation of DNA Template for PCR. DNA templates were prepared according to the previous described method [12]. A $300 \mu \mathrm{L}$ of overnight culture of the test isolates in tryptone soy broth (Difco, Detroit, MI, USA) was centrifuged. The bacterial pellet was resuspended to the initial volume with HPLC grade water. The DNA template was prepared by boiling of suspension of bacterial pellet for $10 \mathrm{~min}$ and directly used in the PCR assay.

2.8. Detection of ESBL and MBL Genes. Genes for ESBLs (OXA-10-like gene, OXA-2-like gene, and VEB) and MBLs (VIM-1, VIM-2, IMP-1, IMP-2, SIM, GIM, SPM, and NDM) were sought by PCR for all isolates using the primers listed in Table 1 according to the previous protocols [7, 13-16]. Negative and positive controls were involved in all PCR experiments. Five $\mu \mathrm{L}$ of reaction mix containing PCR product was analysed by electrophoresis in $0.8 \%(\mathrm{w} / \mathrm{v})$ agarose (Fermentas, Lithuania). 
TABLE 1: Primers used for detection of MBL, OXA 10, and VEB.

\begin{tabular}{|c|c|c|c|}
\hline Primers & Sequence & Reference & Expected PCR product \\
\hline$b l a_{\mathrm{IMP}-1}$ & $\begin{array}{l}\text { TGAGCAAGTTATCTGTATTC } \\
\text { TTAGTTGCTTGGTTTTGATG }\end{array}$ & {$[14]$} & $740 \mathrm{bp}$ \\
\hline$b l a_{\mathrm{IMP}-2}$ & $\begin{array}{l}\text { GGCAGTCGCCCTAAAACAAA } \\
\text { TAGTTACTTGGCTGTGATGG }\end{array}$ & {$[14]$} & $737 \mathrm{bp}$ \\
\hline$b l a_{\mathrm{VIM}-1}$ & $\begin{array}{l}\text { TTATGGAGCAGCAACGATGT } \\
\text { CAAAAGTCCCGCTCCAACGA }\end{array}$ & {$[14]$} & $920 \mathrm{bp}$ \\
\hline$b l a_{\mathrm{VIM}-2}$ & $\begin{array}{l}\text { AAAGTTATGCCGCACTCACC } \\
\text { TGCAACTTCATGTTATGCCG }\end{array}$ & {$[14]$} & $865 \mathrm{bp}$ \\
\hline$b l a_{\mathrm{NDM}}$ & $\begin{array}{c}\text { CACCTCATGTTTGAATTCGCC } \\
\text { CTCTGTCACATCGAAATCGC }\end{array}$ & {$[16]$} & $984 \mathrm{bp}$ \\
\hline$b l a_{\text {OXA-10 }}$ & $\begin{array}{c}\text { TATCGCGTGTCTTTCGAGTA } \\
\text { TTAGCCACCAATGATGCCC }\end{array}$ & {$[7]$} & $760 \mathrm{bp}$ \\
\hline$b l a_{\mathrm{VEB}-1}$ & $\begin{array}{l}\text { CGACTTCCATTTCCCGATGC } \\
\text { GGACTCTGCAACAAATACGC }\end{array}$ & {$[7]$} & $642 \mathrm{bp}$ \\
\hline$b l a_{\text {OXA-2 }}$ & $\begin{array}{l}\text { GCCAAAGGCACGATAGTTGT } \\
\text { GCGTCCGAGTTGACTGCCGG }\end{array}$ & {$[13]$} & $700 \mathrm{bp}$ \\
\hline$b l a_{\mathrm{GIM}}$ & $\begin{array}{c}\text { TCGACACACCTT GGT CTG AA } \\
\text { AACTTCCAACTT TGCCATGC }\end{array}$ & {$[15]$} & $477 \mathrm{bp}$ \\
\hline$b l a_{\mathrm{SPM}}$ & $\begin{array}{c}\text { AAAATCTGGGTACGCAAA CG } \\
\text { ACATTATCCGCTGGAACAGG }\end{array}$ & {$[15]$} & $271 \mathrm{bp}$ \\
\hline$b l a_{\mathrm{SIM}}$ & $\begin{array}{l}\text { TAC AAG GGATTCGGCATCG } \\
\text { TAATGG CCTGT CCCATG TG }\end{array}$ & {$[15]$} & $570 \mathrm{bp}$ \\
\hline
\end{tabular}

TABLE 2: Resistance rates for clinical $P$. aeruginosa isolates.

\begin{tabular}{|c|c|c|c|c|}
\hline \multirow[b]{2}{*}{ Antibiotic } & \multicolumn{4}{|c|}{ Number (\%) of resistant isolates } \\
\hline & $\begin{array}{c}\text { Total isolates } \\
\quad(n=122)\end{array}$ & $\begin{array}{l}\text { Imipenem susceptible } \\
\qquad(n=66)\end{array}$ & $\begin{array}{l}\text { Imipenem intermediate } \\
\qquad(n=8)\end{array}$ & $\begin{array}{c}\text { Imipenem resistant } \\
\quad(n=48)\end{array}$ \\
\hline \multicolumn{5}{|l|}{$\beta$-lactams } \\
\hline Cefuroxime & $107(87.7 \%)$ & $51(77.2 \%)$ & $8(100 \%)$ & $48(100 \%)$ \\
\hline Cefoperazone & $98(80.3 \%)$ & $43(65.2 \%)$ & $8(100 \%)$ & $47(97.9 \%)$ \\
\hline Ceftazidime & $74(60.6 \%)$ & $23(34.8 \%)$ & $8(100 \%)$ & $43(89.5 \%)$ \\
\hline Meropenem & $56(45.9 \%)$ & $3(4.5 \%)$ & $7(87.5 \%)$ & $46(95.8 \%)$ \\
\hline Aztreonam & $55(45.1 \%)$ & $28(42.4 \%)$ & $3(37.5 \%)$ & $24(50 \%)$ \\
\hline Imipenem & $48(39.3 \%)$ & $66(100 \%)$ & $8(100 \%)$ & $48(100 \%)$ \\
\hline Piperacillin/tazobactam & $31(25.4 \%)$ & $4(6.1 \%)$ & $7(87.5 \%)$ & $20(41.6 \%)$ \\
\hline \multicolumn{5}{|l|}{ Non $\beta$-lactams } \\
\hline Gentamicin & $61(50 \%)$ & $13(19.7 \%)$ & $5(62.5 \%)$ & $43(89.5 \%)$ \\
\hline Ciprofloxacin & $53(43.4 \%)$ & $17(25.8 \%)$ & $5(62.5 \%)$ & $31(64.5 \%)$ \\
\hline Amikacin & $40(32.8 \%)$ & $9(13.6 \%)$ & $7(87.5 \%)$ & $24(50 \%)$ \\
\hline Polymyxin B & $3(2.4 \%)$ & $0(0.0 \%)$ & $1(12.5 \%)$ & $2(4.2 \%)$ \\
\hline
\end{tabular}

\section{Results}

The antimicrobial susceptibility testing was done by disc diffusion method to 122 clinical isolates of $P$. aeruginosa that were collected from Kasr El Aini Hospital and National Cancer Institute, Cairo University, Egypt, in the period from January 2011 to March 2012.

The resistant rates of antibiotics are shown in Table 2. Forty-eight (39.34\%) out of 122 P. aeruginosa isolates were resistant to imipenem. Eight (6.5\%) out of 122 isolates of
$P$. aeruginosa showed intermediate resistance to imipenem. Fifty-six (46\%) out of 122 P. aeruginosa isolates were resistant to meropenem. Only two isolates (1.64\%) out of 122 showed intermediate resistance to meropenem. The resistant rates for $\beta$-lactam antibiotics including cefuroxime, cefoperazone, ceftazidime, aztreonam, and piperacillin/tazobactam were $87.7 \%, 80.3 \%, 60.6 \%, 45.1 \%$, and $25.4 \%$, respectively. The resistant rates for non- $\beta$-lactam antibiotics including gentamicin, ciprofloxacin, and amikacin were $50 \%, 43.4 \%$, and $32.8 \%$, respectively. Only $3(2.5 \%)$ out of 122 P. aeruginosa 
isolates were resistant to polymyxin $\mathrm{B}$. The antimicrobial resistance rates were higher for imipenem-resistant than imipenem-susceptible $P$. aeruginosa isolates (Table 2). Non$\beta$-lactams showed higher activity against imipenem-resistant $P$. aeruginosa than $\beta$-lactams.

Use of combined disk method (imipenem, ceftazidime/ imipenem, and ceftazidime + EDTA) for phenotypic production of MBL allowed the detection of 33 of 122 (27\%) P. aeruginosa isolates.

Combined double disc synergy test was applied to detect ESBL in $122 P$. aeruginosa isolates using ceftazidime alone or with clavulanic acid. Of these, only 9 (7.4\%) isolates were positive for production of ESBL. All ESBL-producing $P$. aeruginosa were found to be resistant to ceftazidime. Five out of $33 \mathrm{MBL}$-producing isolates were found to produce ESBL and MBL simultaneously.

The results of MICs for MBL-producing $P$. aeruginosa isolates appeared in Table 3 which showed that the strains have high resistance toward imipenem, cefotaxime, gentamicin, and ciprofloxacin as their MIC was above the break points recommended by CLSI. The effect of clavulanic acid on the susceptibility was found as some isolates showed a decrease in MIC more than 3 doubling dilutions, and that indicates the presence of ESBL. On the other hand, according to the breakpoints recommended by CLSI, more than half of the isolates were sensitive toward piperacillin/tazobactam, ceftazidime, and cefepime and ceftazidime/clavulanic acid.

PCR experiments revealed amplification of $865 \mathrm{bp}$ fragment corresponding to $b l a_{\mathrm{VIM}-2}$-like gene in 28 of 48 (58.3\%) imipenem-resistant isolates and a $760 \mathrm{bp}$ fragment corresponding to $b l a_{\mathrm{OXA}-10}$-like gene in $20(41.5 \%)$ of imipenemresistant isolates and a $642 \mathrm{bp}$ fragment corresponding to bla $a_{\mathrm{VEB}}$ gene in $5(10.4 \%)$ of isolates; two isolates $(4.2 \%)$ showed a fragment of $984 \mathrm{bp}$ corresponding to $b l a_{\mathrm{NDM}}$ gene and only one isolate $(2.1 \%)$ showed a fragment of a $740 \mathrm{bp}$ corresponding to $b l a_{\mathrm{IMP}-1}$. In this work MBL $b l a_{\mathrm{VIM}-1}$, $b l a_{\mathrm{IMP}-2}, b l a_{\mathrm{GIM}}, b l a_{\mathrm{SIM}}$, and $b l a_{\mathrm{SPM}}$ allele were not detected; also OXA-2 like gene was not detected in this study.

OXA-10-like gene was concomitant with VIM-2 and/or VEB. Twelve isolates harbored both OXA-10 and VIM-2; however two isolates carried both OXA-10 and VEB. Only one MBL-producing strain contained OXA-10, VIM-2, and VEB (Table 4).

\section{Discussion}

Carbapenems are among the best choices for the treatment of infections caused by multidrug resistant Gram-negative rods. In recent years, Egypt has been considered among the countries that reported high rates of antimicrobial resistance [17]. In the present study, there were high levels of resistance to all commercially available antimicrobial agents among $P$. aeruginosa isolated from Kasr El Aini Hospital and National Cancer Institute, Cairo University, Egypt; the rate of $39.3 \%$ imipenem-resistant isolates, this rate of carbapenem resistance reflects a threat limiting the treatment options in our hospitals. This can be explained in part by the increase in consumption of antimicrobial agents in the last decade leading to a selective pressure of antibiotics on $P$. aeruginosa and consequently the bacteria modify the resistant mechanisms. A similar high rate of resistance has been reported in many developing countries worldwide [18]. In Egypt, Ashour and El-Sharif [19] concluded that Acinetobacter and Pseudomonas species exhibited the highest resistance levels to imipenem (37.03\%) among other Gram-negative organisms [19]. Also Mahmoud et al. [17] showed that among $P$. aeruginosa strains $33.3 \%$ were resistant to imipenem [17].

In the Middle East the occurrence of imipenem resistant $P$. aeruginosa is alarmingly recognized. In Saudi Arabia, the resistance rate of $P$. aeruginosa to imipenem was increased to $38.57 \%$ in 2011 [20]. Among 33 European countries participating in the European Antimicrobial Resistance Surveillance System in 2007, six countries reported carbapenem resistance rates of $>25 \%$ among $P$. aeruginosa isolates; the highest rate was reported from Greece (51\%) [21].

The clinically important MBL families are located in horizontally transferrable gene cassettes and can be spread among Gram-negative bacteria. Although we have not studied this horizontal transfer in the current study, it has been well demonstrated by several previous reports from other groups. Different families of these enzymes have been reported from several geographical regions so far. The most commonly reported families are IMP (for active on imipenem, first isolated in Japan), VIM (for Verona Integron-encoded metallo- $\beta$-lactamase, first isolated in Italy), GIM (for German Imipenemase), SPM (for Sao Paulo metallo- $\beta$-lactamase, first isolated in Brazil), and SIM (for Seoul Imipenemase, first isolated in Korea). IMP- and VIM-producing Pseudomonas strains have been reported worldwide, in different geographical areas [22]. In the current study $27 \%$ of 122 total $P$. aeruginosa isolates were positive for the production of $\mathrm{MBL}$ based on the results of phenotypic screening for MBL. This was lower than the prevalence of MBL producers in Egyptian study which was $32.3 \%$ [23]. However our finding agreed with an Indian study in which $28.57 \%$ of $P$. aeruginosa was found to produce MBL [24]. In the present study, VIM-2 was the most frequently detectable gene among the different MBL genes investigated; the percent of $58.3 \%$ among imipenem-resistant $P$. aeruginosa was detected.

This finding was supported by results of previous studies demonstrating VIM-2 as the most dominant MBL implicated in imipenem resistant $P$. aeruginosa and confers the greatest clinical threat [25]. Worldwide, VIM-2 is the dominant MBL gene associated with nosocomial outbreaks due to MBLproducing $P$. aeruginosa [26]. In our study, out of $33(27 \%)$ MBL producers, $26(78.8 \%)$ were positive for genes detected by PCR and 15 (31.3\%) out of 48 imipenem resistant isolates were positive for genes investigated by PCR and in the same time were negative MBL producers. This indicated that there are other resistance mechanisms to carbapenem such as class A carbapenemases including KPC and GES variants and MBLs were not the sole mechanism of carbapenem resistance in the present study. The imipenem resistant strain with no phenotypic or genotypic sign of $\mathrm{MBL}$ production may possess other enzyme mediating carbapenem resistance such as AmpC beta lactamase and/or other mechanisms such as membrane permeability and efflux mechanisms. 
TABLE 3: MICs of antibiotics for MBL-producing $P$. aeruginosa isolates.

\begin{tabular}{|c|c|c|c|c|c|c|c|c|c|}
\hline \multirow{3}{*}{ Isolates number } & \multicolumn{9}{|c|}{ MICs $(\mu \mathrm{g} / \mathrm{mL})$} \\
\hline & PM & PTc & $\mathrm{TZ}$ & TZL & CI & $\mathrm{AK}$ & GM & IP & $\mathrm{CT}$ \\
\hline & $\geq 32$ & $\geq 128 / 4$ & $\geq 128 / 2$ & $\geq 32$ & $\geq 4$ & $\geq 64$ & $\geq 4$ & $\geq 16$ & $\geq 32$ \\
\hline 1 & $\geq 256$ & 16 & $\geq 256$ & $\geq 256$ & 2 & 12 & 3 & $\geq 32$ & $\geq 256$ \\
\hline 2 & 12 & 64 & 8 & 3 & $\geq 32$ & 16 & $\geq 256$ & $\geq 32$ & $\geq 256$ \\
\hline 3 & 8 & 64 & 64 & 24 & $\geq 32$ & $\geq 256$ & 12 & $\geq 32$ & $\geq 256$ \\
\hline 4 & 4 & 3 & 6 & 2 & $\geq 32$ & $\geq 256$ & $\geq 256$ & $\geq 32$ & 32 \\
\hline 5 & 4 & 3 & 6 & 1.5 & $\geq 32$ & $\geq 256$ & $\geq 256$ & $\geq 32$ & 32 \\
\hline 6 & 64 & $\geq 256$ & $\geq 256$ & $\geq 256$ & $\geq 32$ & $\geq 256$ & $\geq 256$ & $\geq 32$ & $\geq 256$ \\
\hline 7 & 24 & $\geq 256$ & $\geq 256$ & 192 & $\geq 32$ & $\geq 256$ & 12 & $\geq 32$ & $\geq 256$ \\
\hline 8 & 6 & 96 & 96 & 24 & 0.094 & 4 & 2 & $\geq 32$ & $\geq 256$ \\
\hline 9 & $\geq 256$ & 3 & $\geq 256$ & $\geq 256$ & $\geq 32$ & 32 & 12 & $\geq 32$ & $\geq 256$ \\
\hline 10 & $\geq 256$ & 2 & 4 & 1.5 & $\geq 32$ & 2 & 2 & $\geq 32$ & 16 \\
\hline 11 & $\geq 256$ & $\geq 256$ & $\geq 256$ & $\geq 256$ & $\geq 32$ & $\geq 256$ & 32 & $\geq 32$ & $\geq 256$ \\
\hline 12 & 6 & 24 & 8 & 16 & $\geq 32$ & $\geq 256$ & 1.5 & $\geq 32$ & $\geq 256$ \\
\hline 13 & $\geq 256$ & $\geq 256$ & $\geq 256$ & $\geq 256$ & $\geq 32$ & $\geq 256$ & $\geq 256$ & $\geq 32$ & $\geq 256$ \\
\hline 14 & $\geq 256$ & 24 & $\geq 256$ & $\geq 256$ & 1 & 6 & 2 & $\geq 32$ & $\geq 256$ \\
\hline 15 & 16 & 24 & 16 & 4 & $\geq 32$ & 12 & $\geq 256$ & $\geq 32$ & $\geq 256$ \\
\hline 16 & $\geq 256$ & $\geq 256$ & 48 & 8 & $\geq 32$ & 96 & $\geq 256$ & $\geq 32$ & $\geq 256$ \\
\hline 17 & 6 & 12 & 12 & 2 & $\geq 32$ & 6 & $\geq 256$ & $\geq 32$ & $\geq 256$ \\
\hline 18 & $\geq 256$ & 4 & $\geq 256$ & $\geq 256$ & $\geq 32$ & 96 & 32 & $\geq 32$ & $\geq 256$ \\
\hline 19 & 64 & $\geq 256$ & $\geq 256$ & 96 & 0.094 & 96 & 32 & $\geq 32$ & $\geq 256$ \\
\hline 20 & 8 & 32 & 12 & 2 & $\geq 32$ & 16 & $\geq 256$ & $\geq 32$ & $\geq 256$ \\
\hline 21 & 16 & 16 & 24 & 4 & $\geq 32$ & 48 & $\geq 256$ & $\geq 32$ & $\geq 256$ \\
\hline 22 & 256 & $\geq 256$ & $\geq 256$ & $\geq 256$ & $\geq 32$ & 128 & 32 & $\geq 32$ & $\geq 256$ \\
\hline 23 & $\geq 256$ & $\geq 256$ & $\geq 56$ & 256 & $\geq 32$ & $\geq 256$ & 32 & $\geq 32$ & $\geq 256$ \\
\hline 24 & 8 & 12 & $\geq 256$ & 4 & 0.064 & 8 & $\geq 256$ & $\geq 32$ & $\geq 256$ \\
\hline 25 & $\geq 256$ & $\geq 256$ & $\geq 256$ & 128 & $\geq 32$ & 48 & 32 & $\geq 32$ & $\geq 256$ \\
\hline 26 & $\geq 256$ & $\geq 256$ & $\geq 256$ & $\geq 256$ & $\geq 32$ & $\geq 256$ & $\geq 256$ & $\geq 32$ & $\geq 256$ \\
\hline 27 & 16 & $\geq 256$ & $\geq 256$ & 32 & 0.064 & 8 & 32 & $\geq 32$ & $\geq 256$ \\
\hline 28 & 64 & $\geq 256$ & $\geq 256$ & $\geq 256$ & 0.064 & 32 & 32 & $\geq 32$ & $\geq 256$ \\
\hline 29 & 2 & 4 & 12 & 2 & 0.125 & 4 & 2 & $\geq 32$ & $\geq 256$ \\
\hline 30 & 2 & 4 & 8 & 1.5 & 0.125 & 4 & 2 & $\geq 32$ & $\geq 256$ \\
\hline 31 & 8 & 128 & 6 & 24 & 0.094 & 6 & 2 & $\geq 32$ & $\geq 256$ \\
\hline 32 & 12 & 32 & 2 & 8 & 0.064 & 6 & 2 & $\geq 32$ & $\geq 256$ \\
\hline 33 & 6 & $\geq 256$ & 8 & 3 & $\geq 32$ & 128 & 2 & $\geq 32$ & $\geq 256$ \\
\hline
\end{tabular}

PM: cefepime, PTc: piperacillin/tazobactam, TZL: ceftazidime/clavulanic acid, TZ: Ceftazidime, CI: ciprofloxacin, AK: Amikacin, GM: Gentamicin, IP: Imipenem, CT: Cefotaxime.

Class A ESBLs are typically identified in P. aeruginosa isolates showing resistance to extended-spectrum cephalosporin (ESCs) [27]. Classical ESBLs have evolved from restrictedspectrum class A TEM and SHV $\beta$-lactamases although a variety of non-TEM and non-SHV class A ESBLs have been described such as CTX-M, PER, VEB, GES, and BEL [5] and class D ESBLs derived from narrow-spectrum OXA $\beta$ lactamases are also well known [28]. Structural genes VEB, OXA, and PER types are the most common ESBLs reported in $P$. aeruginosa [7].

In the present study, production of ESBL was detected in only (7.4\%) out of 122 P. aeruginosa isolates. This was much lower than what was found in a study done by Gharib et al. in 2009 in Egypt in which 24.5\% were ESBL producers [29]. In the present study high prevalence of $b l a_{\text {OXA-10 }}$ was detected in imipenem resistant $P$. aeruginosa isolates; twenty of 48 $(41.7 \%)$ isolates resistant to imipenem were OXA-10 positive followed by VEB-1 which was detected in 5 (10.4\%). In a recent study in Iran, most prevalent ESBL genes included OXA-10 (70\%) and PER-1 (50\%) followed by VEB-1 (31.3\%) [30]. This study agreed with our study in the prevalence of OXA-10 in ESBL-producing P. aeruginosa. However VEB type ESBLs were the predominant ESBL reported in $P$. aeruginosa in a number of studies where ESBLs were commonly seen [7, 31]. Phenotypic methods for detection of ESBL are not reliable in $P$. aeruginosa strains and PCR is advisable since only 9 
TABLE 4: Differential relation between genes investigated and phenotypic methods for MBL and ESBL producing $P$. aeruginosa.

\begin{tabular}{|c|c|c|c|c|c|}
\hline Genes investigated & $\begin{array}{c}\text { VIM-2 } \\
(n=28)\end{array}$ & $\begin{array}{l}\text { OXA-10 } \\
(n=20) \\
\end{array}$ & $\begin{array}{c}\text { VEB } \\
(n=5) \\
\end{array}$ & $\begin{array}{c}\mathrm{NDM} \\
(n=2) \\
\end{array}$ & $\begin{array}{l}\text { IMP-1 } \\
(n=1) \\
\end{array}$ \\
\hline \multicolumn{6}{|l|}{$\operatorname{MBL}(n=33)$} \\
\hline Pos. & 21 & 14 & 4 & 0 & 1 \\
\hline Neg. & 7 & 6 & 1 & 2 & 0 \\
\hline \multicolumn{6}{|l|}{$\operatorname{ESBL}(n=9)$} \\
\hline Pos. & 4 & 4 & 1 & 2 & 0 \\
\hline Neg. & 24 & 16 & 4 & 0 & 1 \\
\hline \multicolumn{6}{|l|}{ VIM-2 } \\
\hline Pos. & 28 & 12 & 2 & 0 & 1 \\
\hline Neg. & 0 & 8 & 3 & 2 & 0 \\
\hline \multicolumn{6}{|l|}{ OXA-10 } \\
\hline Pos. & 12 & 20 & 2 & 1 & 0 \\
\hline Neg. & 16 & 0 & 3 & 1 & 1 \\
\hline \multicolumn{6}{|l|}{ VEB } \\
\hline Pos. & 2 & 2 & 5 & 0 & 0 \\
\hline Neg. & 26 & 18 & 0 & 2 & 1 \\
\hline \multicolumn{6}{|l|}{$\mathrm{NDM}$} \\
\hline Pos. & 0 & 1 & 0 & 2 & 0 \\
\hline Neg. & 28 & 19 & 5 & 0 & 1 \\
\hline \multicolumn{6}{|l|}{ IMP-1 } \\
\hline Pos. & 1 & 0 & 0 & 0 & 1 \\
\hline Neg. & 27 & 20 & 5 & 2 & 0 \\
\hline
\end{tabular}

isolates were ESBL producers upon phenotypic screening while 20 isolates were positive OXA-10 and 5 were VEB positive using PCR for their detection.

KPC rarely was detected in $P$. aeruginosa; however the number of reports of KPC-producing $P$. aeruginosa is increasing [32]. In this study, we did not test KPC. KPC and another rarely carbapenemases may be found in ESBL-producing strains because most of them had reduced susceptibility to imipenem (MIC 2-8 mg/L).

In the current study $97.5 \%$ of the total $P$. aeruginosa isolates were sensitive to polymyxin $\mathrm{B}$. This supports the evidence that polymyxin $\mathrm{B}$ has increasingly become the last viable therapeutic option for multidrug resistant (MDR) Pseudomonas infections. This result agreed with a study done by Tawfik el al. in 2012 which they found that all isolates were sensitive to polymyxin [33].

In conclusion, the rates of MBL-producing $P$. aeruginosa and ESBL-producing $P$. aeruginosa isolates from Kasr El Aini Hospital and National Cancer Institute, Cairo University, in Egypt were notable and, unfortunately, only a limited number of antimicrobial drugs are active. Therefore, MBL and ESBL screening should be implemented for routine laboratory studies in routine practice. VIM-2 is the most prevalent MBL producing $P$. aeruginosa in Egypt. OXA-10 is the most prevalent ESBL producing $P$. aeruginosa in Egypt. $\mathrm{MBL}$ is much more prevalent than ESBL as mechanism of resistance in $P$. aeruginosa. Molecular techniques are more reliable than phenotypic screening in detecting ESBL production in $P$. aeruginosa strains. Further studies are needed to specify the most important genes of resistance among $P$. aeruginosa in Egypt.

\section{Conflict of Interests}

The authors declare that there is no conflict of interests regarding the publication of this paper.

\section{Acknowledgments}

The authors extend their appreciation to the Deanship of Scientific Research at King Saud University for funding the work through the research group Project no. RGP-VPP-038.

\section{References}

[1] F. W. Wirth, S. U. Picoli, V. V. Cantarelli et al., "Metallo- $\beta$ lactamase-producing Pseudomonas aeruginosa in two hospitals from Southern Brazil," Brazilian Journal of Infectious Diseases, vol. 13, no. 3, pp. 170-172, 2009.

[2] M.-L. Lambert, C. Suetens, A. Savey et al., "Clinical outcomes of health-care-associated infections and antimicrobial resistance in patients admitted to European intensive-care units: a cohort study," The Lancet Infectious Diseases, vol. 11, no. 1, pp. 30-38, 2011.

[3] E. F. Keen III, B. J. Robinson, D. R. Hospenthal et al., "Prevalence of multidrug-resistant organisms recovered at a military burn center," Burns, vol. 36, no. 6, pp. 819-825, 2010. 
[4] D. K. Mendiratta, V. Deotale, and P. Narang, "Mettallo- $\beta$ lactamase producing Pseudomonas aeruginosa in a hospital from a rural area," Indian Journal of Medical Research, vol. 121, no. 5, pp. 701-703, 2005.

[5] W. H. Zhao and Z. Q. Hu, " $\beta$-lactamases identified in clinical isolates of Pseudomonas aeruginosa," Critical Reviews in Microbiology, vol. 36, no. 3, pp. 245-258, 2010.

[6] J. Wang, J.-Y. Zhou, T.-T. Qu et al., "Molecular epidemiology and mechanisms of carbapenem resistance in Pseudomonas aeruginosa isolates from Chinese hospitals," International Journal of Antimicrobial Agents, vol. 35, no. 5, pp. 486-491, 2010.

[7] A. Mirsalehian, M. Feizabadi, F. A. Nakhjavani, F. Jabalameli, H. Goli, and N. Kalantari, "Detection of VEB-1, OXA-10 and PER-1 genotypes in extended-spectrum $\beta$-lactamase-producing Pseudomonas aeruginosa strains isolated from burn patients," Burns, vol. 36, no. 1, pp. 70-74, 2010.

[8] A. W. Bauer, W. M. Kirby, J. C. Sherris, and M. Turck, "Antibiotic susceptibility testing by a standardized single disk method," American Journal of Clinical Pathology, vol. 45, no. 4, pp. 493496, 1966.

[9] Clinical and Laboratory Standards Institute, Methods for Dilution Antimicrobial Susceptibility Tests for Bacteria That Grow Aerobically: Approved Standard M7-A7, CLSI, Wayne, Pa, USA, 7th edition, 2010.

[10] Y. Glupczynski, P. Bogaerts, A. Deplano et al., "Detection and characterization of class A extended-spectrum- $\beta$-lactamaseproducing Pseudomonas aeruginosa isolates in Belgian hospitals," Journal of Antimicrobial Chemotherapy, vol. 65, no. 5, pp. 866-871, 2010.

[11] D. Bashir, M. A. Thokar, B. A. Fomda et al., "Detection of metallo- $\beta$-lactamase (MBL) producing Pseudomonas aeruginosa at a tertiary care hospital in Kashmir," African Journal of Microbiology Research, vol. 5, no. 2, pp. 164-172, 2011.

[12] S. Lee, Y.-J. Park, M. Kim et al., "Prevalence of Ambler class $\mathrm{A}$ and $\mathrm{D} \beta$-lactamases among clinical isolates of Pseudomonas aeruginosa in Korea," Journal of Antimicrobial Chemotherapy, vol. 56, no. 1, pp. 122-127, 2005.

[13] T. Alipour, N. Sadeghifard, N. Amirmozafari et al., "Incidence of extended spectrum $\beta$-lactamase producing Pseudomonas aeruginosa and frequency of OXA-2 and OXA-10 genes," Australian Journal of Basic and Applied Sciences, vol. 4, no. 8, pp. 3202-3207, 2010.

[14] J.-J. Yan, P.-R. Hsueh, W.-C. Ko et al., "Metallo- $\beta$-lactamases in clinical Pseudomonas isolates in Taiwan and identification of VIM-3, a novel variant of the VIM-2 enzyme," Antimicrobial Agents and Chemotherapy, vol. 45, no. 8, pp. 2224-2228, 2001.

[15] M. J. Ellington, J. Kistler, D. M. Livermore, and N. Woodford, "Multiplex PCR for rapid detection of genes encoding acquired metallo- $\beta$-lactamases," Journal of Antimicrobial Chemotherapy, vol. 59, no. 2, pp. 321-322, 2007.

[16] M. Kaase, P. Nordmann, T. A. Wichelhaus, S. G. Gatermann, R. A. Bonnin, and L. Poirel, "NDM-2 carbapenemase in Acinetobacter baumannii from Egypt," Journal of Antimicrobial Chemotherapy, vol. 66, no. 6, pp. 1260-1262, 2011.

[17] B. A. Mahmoud, A. W. Zahran, R. G. Hindawi, Z. A. Labib, and R. Galal, "Prevalence of multidrug-resistant Pseudomonas aeruginosa in patients with nosocomial infections at a university hospital in Egypt, with special reference to typing methods," Journal of Virology \& Microbiology, vol. 2013, Article ID 290047, 13 pages, 2013.
[18] R. C. Picão, L. Poirel, A. C. Gales, and P. Nordmann, "Diversity of $\beta$-lactamases produced by ceftazidime-resistant Pseudomonas aeruginosa isolates causing bloodstream infections in Brazil," Antimicrobial Agents and Chemotherapy, vol. 53, no. 9, pp. 3908-3913, 2009.

[19] H. M. Ashour and A. El-Sharif, "Species distribution and antimicrobial susceptibility of gram-negative aerobic bacteria in hospitalized cancer patients," Journal of Translational Medicine, vol. 7, article 14, pp. 1-13, 2009.

[20] A. A. Mohamed, A. M. Shibl, S. A. Zaki, and A. F. Tawfik, "Antimicrobial resistance pattern and prevalence of metallo$\beta$-lactamases in Pseudomonas aeruginosa from Saudi Arabia," African Journal of Microbiology Research, vol. 5, no. 30, pp. 55285533, 2011.

[21] M. Souli, I. Galani, and H. Giamarellou, "Emergence of extensively drug-resistant and pandrug-resistant gram-negative bacilli in Europe," Euro Surveillance, vol. 13, no. 47, pp. 1-11, 2008.

[22] M. A. Bahar, S. Jamali, and A. Samadikuchaksaraei, "Imipenemresistant Pseudomonas aeruginosa strains carry metallo- $\beta$ lactamase gene bla $\mathrm{VIM}_{\mathrm{M}}$ in a level I Iranian burn hospital," Burns, vol. 36, no. 6, pp. 826-830, 2010.

[23] S. A. Mansour, O. Eldaly, A. J. Fatani, M. L. Mohamed, and E. M. Ibrahim, "Epidemiological characterization of $P$. aeruginosa isolates of intensive care units in Egypt and Saudi Arabia," Eastern Mediterranean Health Journal, vol. 19, no. 1, pp. 71-80, 2013.

[24] A. S. De, S. H. Kumar, and S. M. Baveja, "Prevalence of metallo- $\beta$-lactamase producing Pseudomonas aeruginosa and Acinetobacter species in intensive care areas in a tertiary care hospital," Indian Journal of Critical Care Medicine, vol. 14, no. 4, pp. 217-219, 2010.

[25] T. R. Walsh, H. Li, M. A. Toleman, P. M. Bennett, and R. N. Jones, "Complete sequence of p07-406, a 24,179-base-pair plasmid harboring the $b l a_{\mathrm{VIM}-7}$ metallo- $\beta$-lactamase gene in a Pseudomonas aeruginosa isolate from the United States," Antimicrobial Agents and Chemotherapy, vol. 52, no. 9, pp. 3099-3105, 2008.

[26] J. Elias, C. Schoen, G. Heinze et al., "Nosocomial outbreak of VIM-2 metallo- $\beta$-lactamase-producing Pseudomonas aeruginosa associated with retrograde urography," Clinical Microbiology and Infection, vol. 16, no. 9, pp. 1494-1500, 2010.

[27] D. Hocquet, P. Plésiat, B. Dehecq, P. Mariotte, D. Talon, and X. Bertrand, "Nationwide investigation of extendedspectrum $\beta$-lactamases, metallo- $\beta$-lactamases, and extendedspectrum oxacillinases produced by ceftazidime-resistant $P$ seudomonas aeruginosa strains in France," Antimicrobial Agents and Chemotherapy, vol. 54, no. 8, pp. 3512-3515, 2010.

[28] L. Poirel, T. Naas, and P. Nordmann, "Diversity, epidemiology, and genetics of class D $\beta$-lactamases," Antimicrobial Agents and Chemotherapy, vol. 54, no. 1, pp. 24-38, 2010.

[29] M. A. Gharib, N. H. Ouda, M. Saeed, L. Hamed, and S. M. Abdel Rahman, "Prevalence of extended spectrum beta lactamase producers in an Egyptian Critical Care Center," Egyptian Journal of Medical Microbiology, vol. 18, no. 4, pp. 139145, 2009.

[30] F. Jabalameli, A. Mirsalehian, N. Sotoudeh et al., "Multiplelocus variable number of tandem repeats (VNTR) fingerprinting (MLVF) and antibacterial resistance profiles of extended spectrum beta lactamase (ESBL) producing Pseudomonas aeruginosa among burnt patients in Tehran," Burns, vol. 37, no. 7, pp. 1202-1207, 2011. 
[31] F. Shahcheraghi, V.-S. Nikbin, and M. M. Feizabadi, "Prevalence of ESBLs genes among multidrug-resistant isolates of Pseudomonas aeruginosa isolated from patients in Tehran," Microbial Drug Resistance, vol. 15, no. 1, pp. 37-39, 2009.

[32] D. J. Wolter, N. Khalaf, I. E. Robledo et al., "Surveillance of carbapenem-resistant Pseudomonas aeruginosa isolates from Puerto Rican Medical Center Hospitals: dissemination of KPC and IMP-18 $\beta$-lactamases," Antimicrobial Agents and Chemotherapy, vol. 53, no. 4, pp. 1660-1664, 2009.

[33] A. F. Tawfik, A. M. Shibl, M. A. Aljohi, M. A. Altammami, and M. H. Al-Agamy, "Distribution of Ambler class A, B and D $\beta$ lactamases among Pseudomonas aeruginosa isolates," Burns, vol. 38, no. 6, pp. 855-860, 2012. 

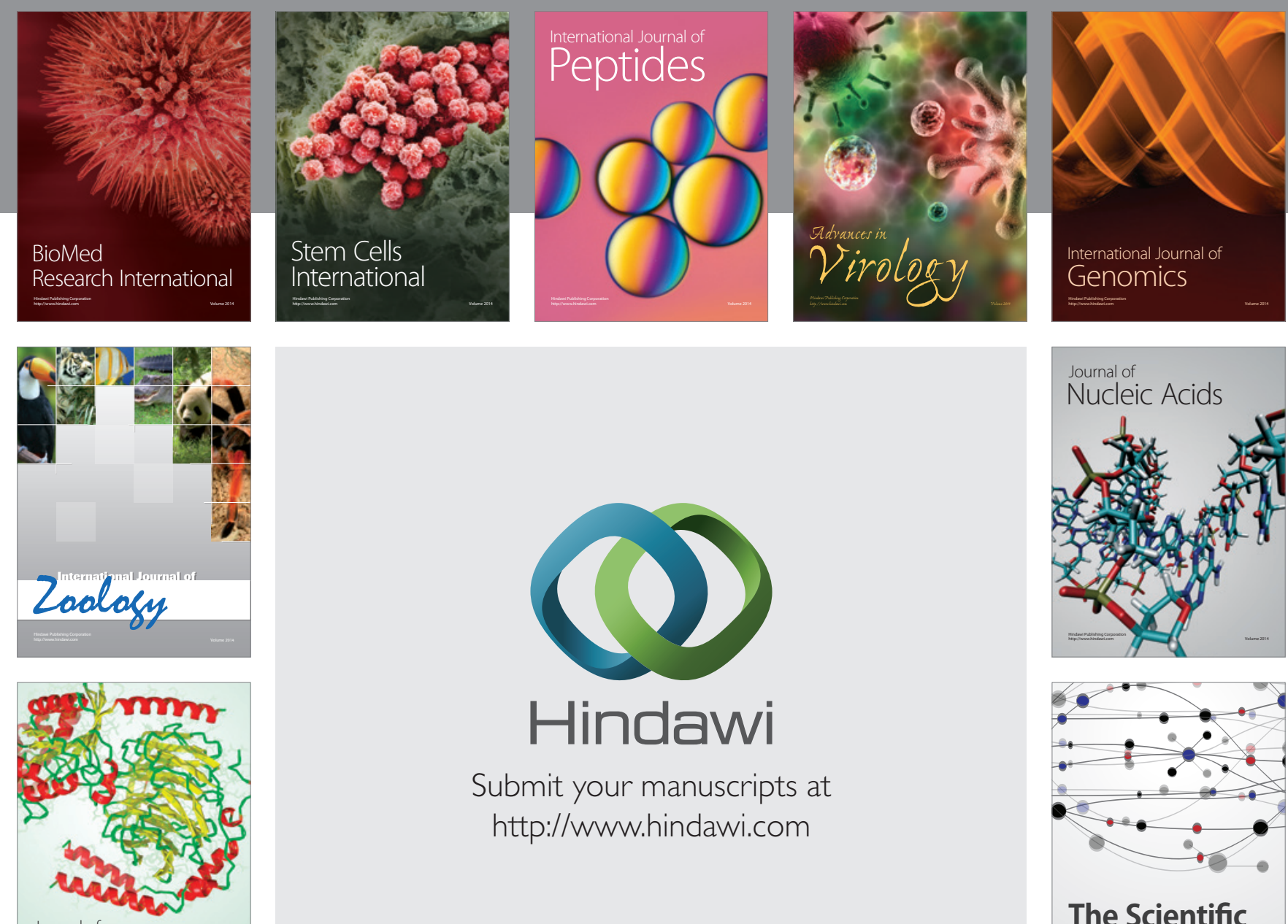

Submit your manuscripts at

http://www.hindawi.com

Journal of
Signal Transduction
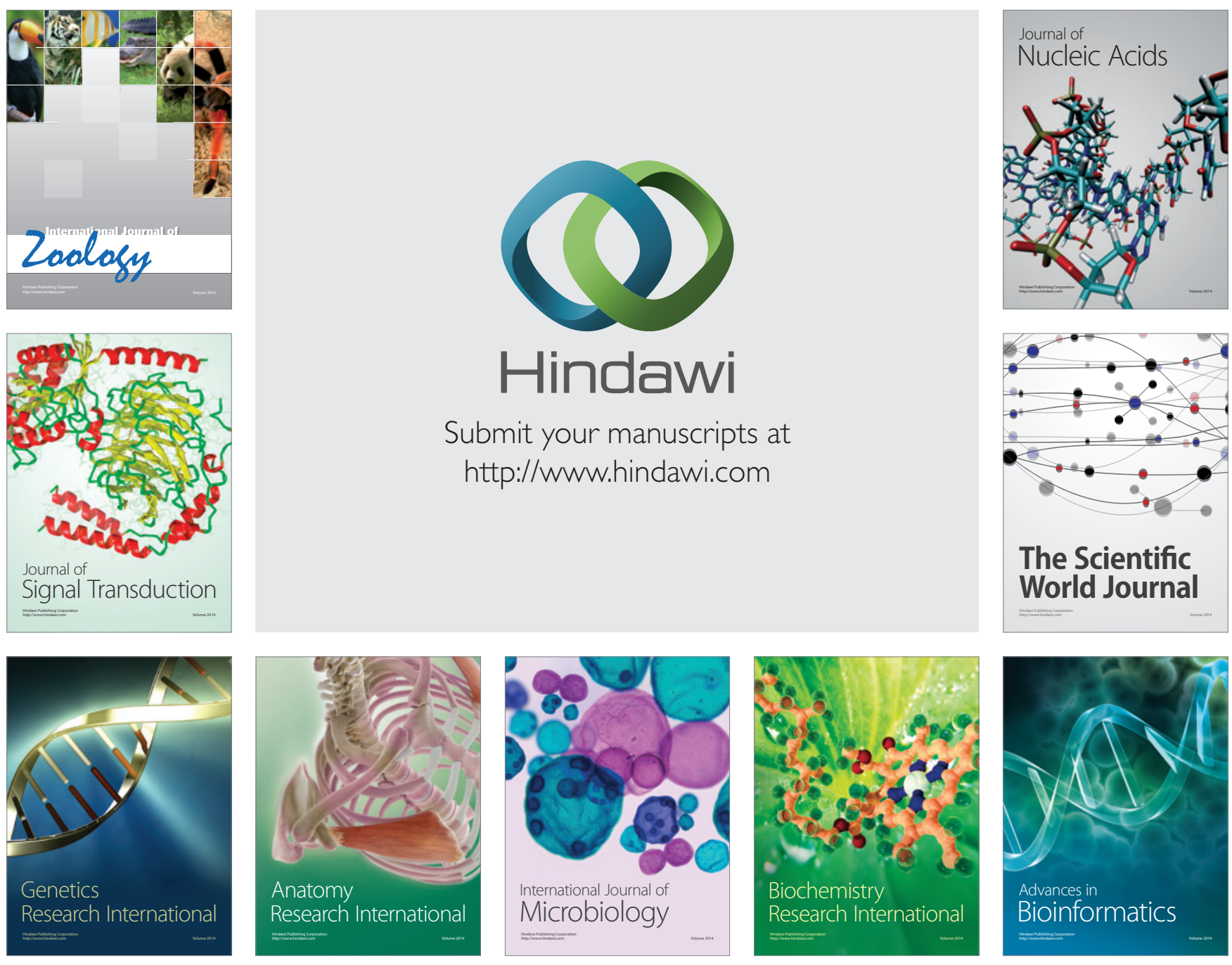

The Scientific World Journal
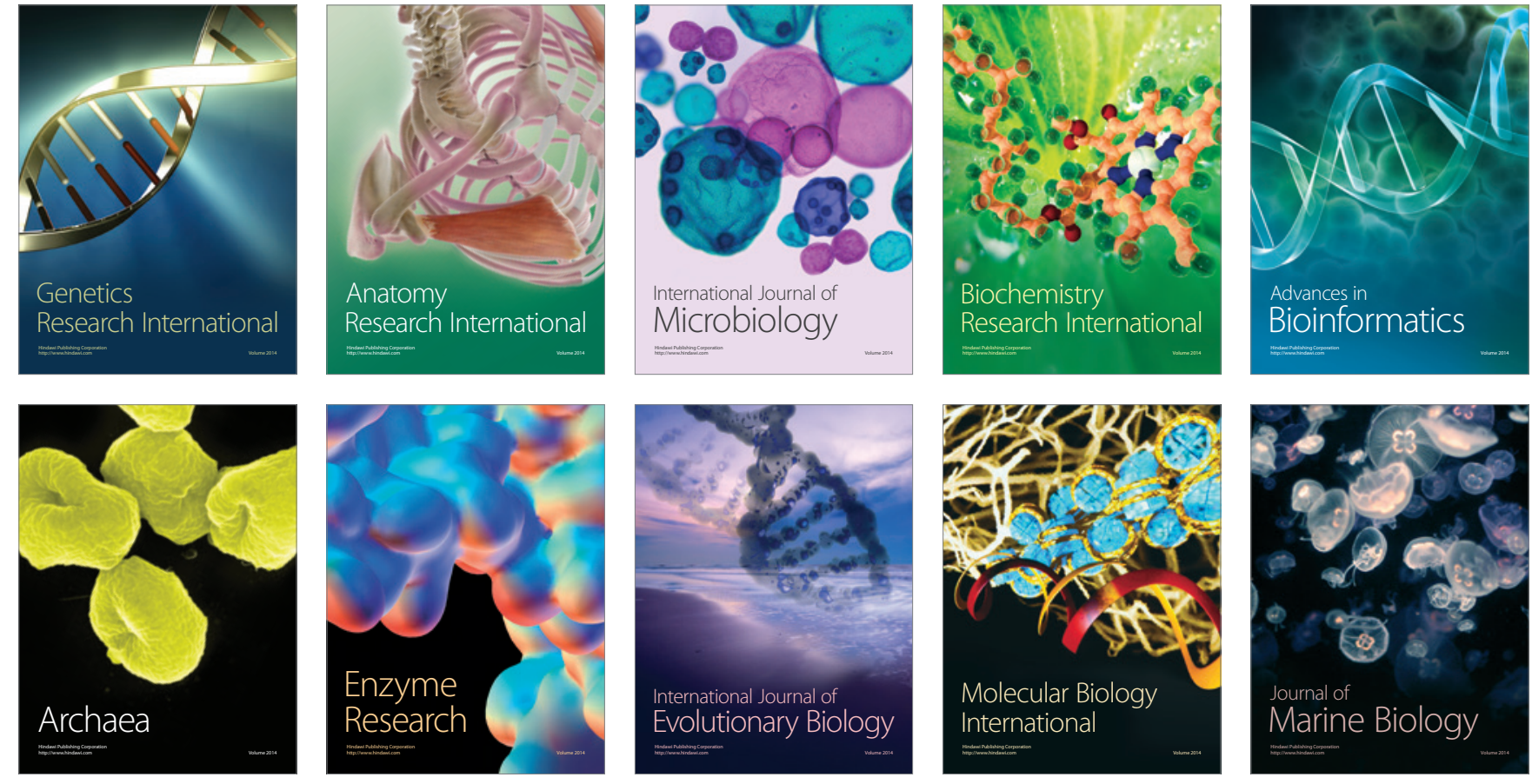